\title{
Erratum
}

\section{An Improved Synthesis of 1,4,7-Triazacyclononanes (tacns) and 1,4,7,10-Tetraazacyclododecanes (cyclens)}

\author{
Jianying Huang, Zhongyuan Zhou, Tak Hang Chan* Synthesis 2009, 2341.
}

In the reaction of compound 7 with ethylenediamine (13) according to Scheme 4, it was reported that 1-tosyl-1,4,7triazacyclononane (9) was obtained in $78 \%$ isolated yield. However, after being alerted by other laboratories, we repeated the same reaction under identical experimental conditions and did not obtain compound $\mathbf{9}$ as the product of the reaction. While the compound obtained was isomeric with 9 according to its mass spectrum, its ${ }^{1} \mathrm{H}$ NMR spectrum was similar but not identical to that of $\mathbf{9}$, a known compound which could be obtained by an alternate route and independently synthesized according to Scheme $2 .{ }^{1}$ More significantly, the ${ }^{13} \mathrm{C}$ NMR spectrum of the obtained product has four aromatic and five aliphatic carbon signals and is distinctly different from that of 1-tosyl1,4,7-triazacyclononane (9) which has eight carbon signals. The product is assigned to have the structure 1-(2'aminoethyl)-4-tosylpiperazine (23) (revised Scheme 4). The formation of a six-membered ring is consistent with the reaction of $\mathbf{7}$ with $N, N$-dimethylethylenediamine (10) in giving 1-methyl-4-tosylpiperazine (11) as we had reported in Scheme 3.

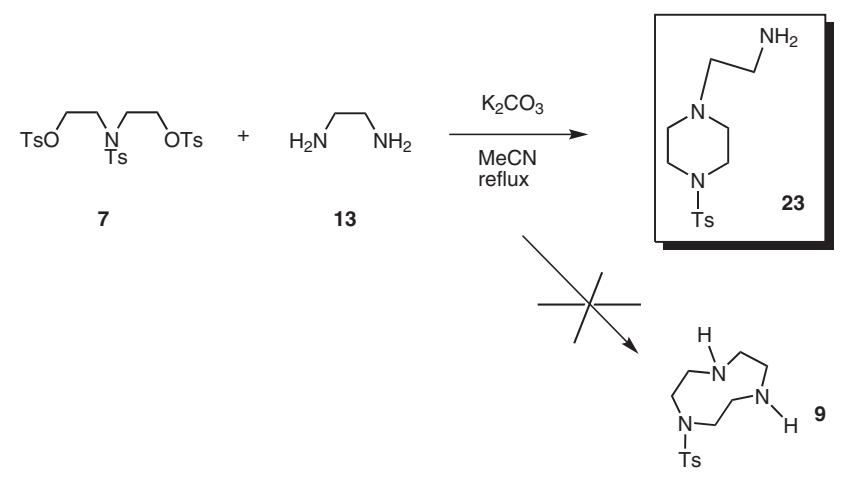

Revised Scheme 4: Reaction of 7 with 13

We also re-examined the coupling of 7 with 1,4,7-triazaheptane (19) using potassium carbonate in refluxing acetonitrile (Scheme 7). The product obtained was found not to be 1-tosyl-1,4,7,10-tetraazacyclododecane (20), a known compound independently synthesized by an alter- nate route. ${ }^{2}$ While the product was isomeric with 20 according to its mass spectrum, its ${ }^{1} \mathrm{H}$ and ${ }^{13} \mathrm{C}$ NMR spectra were different from those of $\mathbf{2 0}$. The product is consistent with a piperazine structure $\mathbf{2 4}$, with four aromatic and six aliphatic carbon signals in its ${ }^{13} \mathrm{C}$ NMR spectrum (revised Scheme 7).

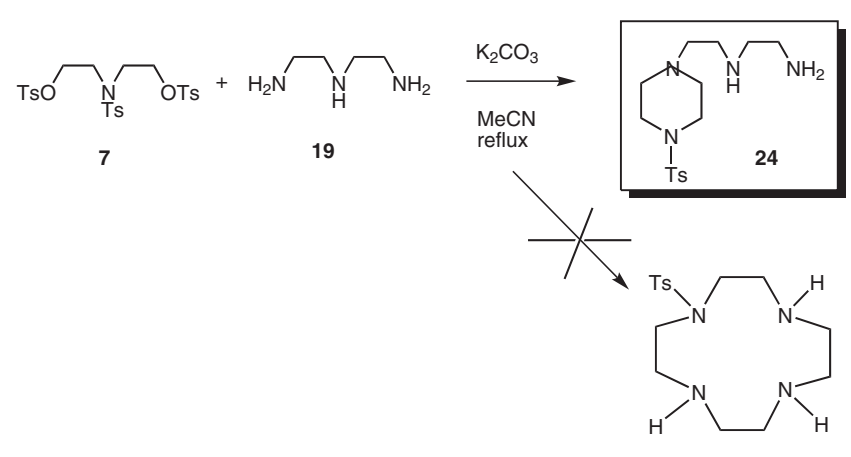

20

Revised Scheme 7: Reaction of 7 with 19

In conclusion, ethylenediamine (13) and 1,4,7-triazaheptane (19) did react with 7 but did not give the corresponding tacn $\mathbf{9}$ or cyclen $\mathbf{2 0}$.

\section{1-(2'-Aminoethyl)-4-tosylpiperazine (23)}

Compound 7 (5.83 g, $10.0 \mathrm{mmol}), \mathrm{K}_{2} \mathrm{CO}_{3}(8.00 \mathrm{~g}, 58.0 \mathrm{mmol})$, ethylenediamine $(0.60 \mathrm{~g}, 10.0 \mathrm{mmol})$ and anhydrous $\mathrm{MeCN}(50 \mathrm{~mL})$ were added to a round-bottom flask. The mixture was heated to reflux under an $\mathrm{N}_{2}$ atmosphere for $12 \mathrm{~h}$. The mixture was cooled to r.t. and filtered. The filtrate was concentrated and the residue was purified by flash chromatography $\left(\mathrm{SiO}_{2}, \mathrm{CH}_{2} \mathrm{Cl}_{2}-\mathrm{MeOH}-\mathrm{Et}_{3} \mathrm{~N}=\right.$ 2:1:0.05 as eluent) to give 23 as a pale yellow oil $(2.0 \mathrm{~g}, 78 \%)$.

${ }^{1} \mathrm{H}$ NMR (400 MHz, $\left.\mathrm{CDCl}_{3}\right): \delta=7.63(\mathrm{~d}, J=7.4 \mathrm{~Hz}, 2 \mathrm{H}), 7.32(\mathrm{~d}$, $J=7.4 \mathrm{~Hz}, 2 \mathrm{H}$ ), 3.01 (br, $4 \mathrm{H}), 2.73(\mathrm{t}, J=6.0 \mathrm{~Hz}, 2 \mathrm{H}), 2.52$ (br t, $4 \mathrm{H}), 2.43-2.40$ (m, $5 \mathrm{H})$.

${ }^{13} \mathrm{C}$ NMR $\left(100 \mathrm{MHz}, \mathrm{CDCl}_{3}\right): \delta=143.7,132.4,129.6,127.8,60.2$, 52.2, 46.0, 38.4, 21.5.

LRMS (ESI): $m / z=284\left(\left[\mathrm{M}^{+}+\mathrm{H}\right], 100\right)$.

HRMS (ESI): calcd for $\mathrm{C}_{13} \mathrm{H}_{22} \mathrm{~N}_{3} \mathrm{O}_{2} \mathrm{~S}\left(\mathrm{M}^{+}+\mathrm{H}\right)$; 284.1433; found: 284.1423. 


\section{1-(1',4'-Diazahexyl)-4-tosylpiperazine (24)}

1,4,7-Triazaheptane $(\mathbf{1 9}, 0.40 \mathrm{~g}, 4.00 \mathrm{mmol})$, compound 7 (2.30 g, $4.00 \mathrm{mmol}), \mathrm{K}_{2} \mathrm{CO}_{3}(6.00 \mathrm{~g}, 40.0 \mathrm{mmol})$ and anhydrous $\mathrm{MeCN}(20$ $\mathrm{mL}$ ) were added to a round-bottom flask. The mixture was heated to reflux under an $\mathrm{N}_{2}$ atmosphere for $18 \mathrm{~h}$. The mixture was cooled to r.t. and filtered. The filtrate was concentrated and the residue was purified by chromatography $\left(\mathrm{SiO}_{2}, \mathrm{CH}_{2} \mathrm{Cl}_{2}-\mathrm{MeOH}=2: 1\right.$ as eluent $)$ to give a light yellow oil $(1.2 \mathrm{~g}, 83 \%)$.

${ }^{1} \mathrm{H} \mathrm{NMR}\left(400 \mathrm{MHz}, \mathrm{CDCl}_{3}\right): \delta=7.58(\mathrm{~d}, J=8.0 \mathrm{~Hz}, 2 \mathrm{H}), 7.27(\mathrm{~d}$, $J=8.0 \mathrm{~Hz}, 2 \mathrm{H}), 2.95(\mathrm{br}, 4 \mathrm{H}), 2.70(\mathrm{t}, J=6.0 \mathrm{~Hz}, 2 \mathrm{H}), 2.59$ (p, $J=6.0 \mathrm{~Hz}, 2 \mathrm{H}), 2.47$ (br t, $4 \mathrm{H}), 2.43(\mathrm{t}, J=6.0 \mathrm{~Hz}, 2 \mathrm{H}), 2.37$ (s, 3 $\mathrm{H})$.

${ }^{13} \mathrm{C} \mathrm{NMR}\left(100 \mathrm{MHz}, \mathrm{CDCl}_{3}\right): \delta=143.7,132.4,129.6,127.8,57.4$, 52.3, 52.2, 46.1, 46.0, 41.4, 21.5.

LRMS (ESI): $m / z=327\left(\left[\mathrm{M}^{+}+\mathrm{H}\right], 100\right), 349\left(\left[\mathrm{M}^{+}+\mathrm{Na}\right], 29\right)$.

HRMS (ESI): calcd for $\mathrm{C}_{15} \mathrm{H}_{27} \mathrm{~N}_{4} \mathrm{O}_{2} \mathrm{~S}\left[\mathrm{M}^{+}+\mathrm{H}\right]: 327.1855$; found: 327.1856 .

\section{Acknowledgment}

We gratefully acknowledge the help of Dr. Kin-Fai Chan for repeating the experiments.

\section{References}

(1) (a) Flassbeck, C.; Wieghardt, K. Z. Anorg. Allg. Chem. 1992, 608, 60. (b) Romakh, V. B.; Therrien, B.; Labat, G.; Stoekli-Evans, H.; Shul'pin, G. B.; Suss-Fink, G. Inorg. Chim. Acta 2006, 359, 3297. (c) Romakh, V. B.; Therrien, B.; Karmazin-Brelot, L; Labat, G.; Stoekli-Evans, H.; Shul'pin, G. B.; Suss-Fink, G. Inorg. Chim. Acta 2006, 359, 1619. (d) Bambirra, S.; Leusen, D. V.; Cornelis G. J.; Tazelaar, A. M.; Hessen, B. Organometallics 2007, 26, 1014.

(2) Compound 20 could be obtained by mono-tosylation of commercially available 2. See: (a) Ohashi, M.; Konkol, M.; Del Rosal, I.; Poteau, R.; Maron, L.; Okuda, J. J. Am. Chem. Soc., 2008, 130, 6920. (b) Leivers, M.; Breslow, R. Bioorg. Chem., 2001, 29, 345. 